\title{
El proyecto FEM-CELL: Círculos autogestivos de mujeres para el aprendizaje de la lengua inglesa The FEM-CELL project: female peer-led circles for English language learning
}

\author{
Nathalie Vázquez Monter ${ }^{1}$, Agustín Solano ${ }^{2}$, Mariana Escobar ${ }^{3}$ \\ nathalie.v.monter@gmail.com, solano.lopez.agustin@gmail.com, marianaescobar215@gmail.com \\ ${ }^{1,3}$ Investigación y Proyectos Educativos \\ Hipatia México \\ Tequisquiapan, México \\ ${ }^{2}$ Facultad de Filosofía y Letras \\ Universidad Nacional Autónoma de México \\ Ciudad de México, México
}

\begin{abstract}
Resumen-. El Proyecto FEM-CELL se plantea como una propuesta innovadora para la conformación de círculos autogestivos de mujeres, enfocado al aprendizaje de la lengua inglesa. El trabajo que aquí se presenta reporta los primeros resultados al implementar el programa en una fase de piloto de seis meses tanto en México como en Colombia. Los resultados señalan las debilidades y fortalezas de este programa voluntario y gratuito, y explora algunos conceptos de pedagogías descolonizadoras y feministas. Por último, los resultados mencionan las adecuaciones que deberán promoverse para garantizar el logro de los objetivos de aprendizaje y el desarrollo personal de las participantes. El proyecto al término de la primera etapa piloto, podrá sentar las bases para su escalamiento en una segunda etapa.
\end{abstract}

Palabras clave: tecnología educativa, círculos de aprendizaje autogestivos, aprendizaje del idioma inglés

\begin{abstract}
The FEM-CELL project is an innovative proposal for the creation of female peer-led circles focused on English language learning. The work presented here reports the first results after implementing the programme in a six-month pilot phase in both Mexico and Colombia. In this sense, the results point out the weaknesses and strengths of this voluntary and free programme, but also lay the foundations of a theoretical framework that critical pedagogies such as popular, decolonising and feminist pedagogies contribute to the proposal. Finally, the results mention the adaptations that should be promoted to guarantee the achievement of learning objectives and the personal development of the participants. At the end of the first pilot stage, the project will be able to scale up in a second stage.
\end{abstract}

Keywords: educational technology, peer-led learning circles, English language learning

\section{INTRODUCCIÓN}

La situación social y económica de los últimos diez años en México y Colombia ha tenido un impacto negativo en la calidad de la educación tanto pública como privada recibida por niñas, niños, adolescentes y mujeres en ambos países (Santiago, Paulo; Isobel McGregor, Deborah Nusche, Pedro Ravela y Diana Toledo: 2012; Arreola Medina, Gonzalo: 2014). Esto ha afectado directamente la asimilación de contenidos educativos por parte de las estudiantes; pero indirectamente y más importante aún, es que ha afectado su desarrollo personal (Argüello Cabrera, 2016; Solano López y Trujillo Reyes, 2021).
La emergencia sanitaria por Covid-19 agravó dicha situación en los últimos dos años, degradando significativamente la calidad educativa $y$ poniendo al descubierto la ausencia de infraestructura tecnológica, al mismo tiempo que se evidenció la necesidad de programas de formación docente integral y continua, colegiada y tutorada. Con este proyecto se presenta una alternativa educativa extra escolar, enfocada en fomentar el desarrollo integral de adolescentes y mujeres a través de la conformación de grupos autogestivos para el aprendizaje de la lengua inglesa.

La propuesta, iniciada por las tutoras, fue inspirada en la propuesta de Shinoda-Bolen, expresa en el "Millonésimo Círculo", una alternativa que permita revolucionar la manera en la que los grupos sociales se organizan y gestionan con el fin de generar cambios evolutivos en la organización a nivel de especie (Shinoda-Bolen, 2008). Ella plantea, que a través de la multiplicación de "círculos sagrados" de mujeres, es posible desarrollar una forma segura, democrática, consciente y solidaria de participar en la decisión de grupos. Para la autora, es en los círculos de mujeres en donde se gesta la posibilidad de conformar círculos sagrados, los cuales deberán permitir a las participantes desarrollar sus habilidades socio-emocionales tales como la escucha atenta y la empatía para el desarrollo de todo el grupo. De este modo, Shinoda-Bolen establece una guía práctica sobre estrategias para el desarrollo socio-emocional de las participantes.

Debo advertir que el tema principal del artículo no permitiría la definición de un estado de arte de los círculos autogestivos a detalle, situación además difícil porque tendría que corresponder al estado de emergencia sanitaria, cuya influencia data de los últimos dos años. Sin embargo, es útil mencionar algunas referencias sobre casos anteriores que han brindado luz e inspiración sobre el desarrollo de estos círculos. Tal es el caso del proyecto del Consejo Británico denominado EDGE (English and Digital for Girl's Education), cuyo objetivo también establece el aprendizaje del inglés a través de círculos autogestivos de mujeres (British Council, 2012).

Las aportaciones de esta propuesta consideramos que son principalmente dos. Primero, una contribución al estado del arte sobre el estudio y desarrollo de nuevos modelos educativos, ante la urgente necesidad de adaptarse rápidamente a las 
necesidades educativas, las condiciones económicas y a la infraestructura tecnológica disponible, especialmente ante la situación de emergencia sanitaria por COVID-19 en países como México y Colombia. El aporte se concreta en el desarrollo de un programa que resulte pertinente a las diferentes condiciones de acceso y disponibilidad de infraestructura tecnológica y cultural de los círculos. El programa consiste fundamentalmente en la descripción de dos modelos didácticos, basados en el desarrollo de habilidades de autogestión, propuestas por las pedagogías populares y descolonizadoras (Freire, 1972; Corvalán, G., Di Matteo et al., 2018).

La segunda, y quizás la más relevante, es la aportación al estado del arte en el ámbito concreto del desarrollo de habilidades para la gestión del aprendizaje, tanto en un plano individual como colectivo. La propuesta expresa la necesidad de instrumentar de manera prioritaria procesos de enseñanzaaprendizaje que permitan a las participantes prescindir eventualmente de la tutora, a la vez que se consolida el funcionamiento de un entorno adecuado e íntegro.

\section{CONTEXTO}

\section{A. Necesidades identificadas}

Tanto en México como en Colombia, la idea de proporcionar cursos de inglés, se consideró a partir de la necesidad que diferentes mujeres manifestaron en las redes sociales. Una vez conformados los grupos, el interés por proveer a las participantes de las herramientas para la autogestión, surgió de las tutoras, posteriormente adoptado por Hipatia México. De esta manera, el inglés resultó ser el agente motivador del aprendizaje.

Las participantes fueron mujeres voluntarias y que pudieran contar con los recursos tecnológicos para tomar las sesiones. Nadie predeterminó la estructura o funcionamiento de los círculos. Esto quedó determinado por las participantes a partir de sus características y necesidades. Además, los grupos fueron itinerantes, es decir: las participantes podían entrar y salir a su gusto. Dado que la iniciativa surgió a través de las redes, se decidió desarrollar el programa en línea, dándole mayor cobertura, en las condiciones de emergencia sanitaria que prevalecían en ambos países. Esto permitió que la ubicación geográfica de las tutoras y de las participantes no fuera un aspecto importante a considerar. Por ejemplo, mientras la tutora de los grupos en Colombia, se ubicaba en la capital de dicho país, la tutora de los grupos en México se ubicaba fuera de México.

\section{B. Objetivos}

El objetivo general de este proyecto es la conformación y consolidación de círculos autogestivos de mujeres en México y Colombia enfocados en el cuidado y fortalecimiento de la agencia de diferentes mujeres que, a pesar de estar inmersas en un contexto de violencia sistémica, puedan desarrollar liderazgos y grupos autogestionados de aprendizaje colectivo tanto de temáticas de interés como de la lengua inglesa.

En estos círculos autogestivos de mujeres se llevaron a cabo actividades sugeridas por las mismas participantes, que fueran significativas para su contexto y que las vinculara con la familia, el espacio escolar, la comunidad y su vida cotidiana. Los espacios de trabajo sostenidos por estos grupos de mujeres ofrecieron un proceso de construcción de diálogo y de esperanza para lograr su desarrollo pleno.
Como parte del objetivo del proyecto no pretendemos determinar un currículum único, replicable para los diferentes contextos en los que se aplica, sino por el contrario, construirlo de manera orgánica; es decir, a partir de la continua retroalimentación de las participantes y la promoción de su participación activa. Eventualmente, el objetivo último es que el círculo se consolide de manera autogestiva; y pretender en un corto plazo, el desarrollo de un modelo de currículum, lo suficientemente flexible, para adaptarse a los diversos escenarios tecnológicos y culturales existentes.

Los cinco objetivos específicos del proyecto, acordados inicialmente por las tutoras, se enumeran a continuación:

1. Promover el desarrollo de las habilidades comunicativas y socio-emocionales de las participantes de tal manera que les permita perder el miedo a expresarse en inglés independientemente del vocabulario L2 que conozcan.

2. Promover, atender y facilitar un proceso constante de formación colectiva y auto formación en temáticas del interés de las participantes, con enfoque en el aprendizaje del idioma inglés.

3. Consolidar espacios que faciliten la construcción de redes de apoyo entre mujeres y/o el fortalecimiento de relaciones interpersonales entre las mismas.

4. Apoyar la sanación de conflictos y traumas previos relacionados con el aprendizaje de inglés.

5. Ofrecer contenidos a los que usualmente las participantes no pueden acceder de manera gratuita.

\section{Preguntas de Investigación}

Para esta propuesta, se planteó una pregunta de investigación principal: ¿Qué estrategias/prácticas fortalecen la autogestión del aprendizaje colectivo en los círculos de mujeres y cómo?

De la pregunta principal anterior, se derivaron las siguientes preguntas específicas de investigación:

1. ¿Qué tipo de actividades y materiales fomentan la autogestión del aprendizaje colectivo?

2. ¿Cómo apoyan las pedagogías populares, descolonizadoras y feministas a este tipo de trabajo colaborativo?

3. ¿Cuáles son los diferentes usos de la tecnología pertinentes para promover el aprendizaje colectivo de manera virtual?

4. ¿Cuáles son las fortalezas y debilidades de esta propuesta?

\section{Escenarios Tecnológicos Latinoamericanos}

Los tres casos que componen el estudio, fueron determinados en un primer momento de acuerdo al país en donde se iniciaron. De esta manera se establecieron dos casos: El Caso I (en Colombia) y el Caso II (en México), integrado por mujeres correspondientes a varias regiones de cada país. En estos dos casos, las participantes tuvieron disponibilidad y acceso a tecnología y conexión continua al internet. El Caso III, surgió posteriormente, al presentarse la oportunidad de extender la propuesta a una escuela comunitaria rural en el Estado de Guerrero en México. En este caso, las condiciones de infraestructura impidieron a las participantes integrarse al Caso II (en el mismo país). Esta definición ya no obedeció al primer criterio de clasificación, sino a una clasificación en función del escenario tecnológico. Sin embargo, es fundamental recordar 
que la intención del proyecto no es determinar un currículum aplicable a los diferentes círculos, sino determinar una metodología que permita adaptar el currículum a necesidades específicas de diversos contextos (modelo) y promover que sean las participantes quienes sean las responsables de desarrollarlo al interior de cada círculo.

Las características generales de las participantes y de los recursos tecnológicos disponibles en los círculos, se especifican a continuación de acuerdo a dos escenarios tecnológicos identificados:

En el primer escenario tecnológico, se encuentran los casos I y II. En el caso de I (Colombia), se conformaron tres grupos (17, 25 y 29 participantes) de acuerdo con su nivel de desempeño: Básico, Intermedio y Avanzado. Por su parte, en el caso II, se conformó sólo un grupo inicialmente con 60 participantes con edades entre los 18 y los 70 años. Este grupo, se dividió posteriormente en dos grupos de acuerdo al nivel de inglés: Elemental y Pre-intermedio. Todas las participantes tuvieron acceso continuo a internet que les permitió el acceso a la plataforma de Classroom de Google, uso de Zoom, Google Meets, Drive, correo electrónico y grupo de Whatsapp.

En el segundo escenario, se encuentra el Caso III, que consistió en un grupo de ocho estudiantes de una escuela Telesecundaria con edades entre los 13 y los 15 años. Las estudiantes viven en una comunidad rural con escasos recursos e infraestructura tecnológica deficiente. No todas las participantes tienen dispositivo personal móvil, ni acceso a internet; por lo que se reúnen en un espacio común con conexión durante una sesión de dos horas por semana. El servicio de internet es con pre-pago y el servicio es intermitente, dado que el lugar de trabajo es la plaza pública de la comunidad. Por otro lado, las estudiantes utilizan un solo teléfono celular para establecer la comunicación entre la tutora y el resto del grupo.

\section{E. Acuerdos para la autogestión}

Aunque los acuerdos para la autogestión de los círculos fueron diferentes para cada uno de ellos, a continuación, se reúnen acuerdos mínimos comunes a todos:

- Las temáticas son propuestas y seleccionadas por las mismas participantes a través de un proceso de votación.

- Se motiva y orienta a las participantes a proponer también las actividades de aprendizaje.

- Mantener un ambiente que propicie la confianza y seguridad de las demás, por lo que se evitan interrupciones para corregir y se abre un espacio de recomendaciones y retroalimentación al final de la (o las) intervenciones.

- Discutir desde el respeto mutuo, enfocándose en las ideas expresadas sin realizar comentarios negativos acerca de las características identitarias o personales de las integrantes ni de su manejo del idioma.

- Apagar el micrófono cuando no se está hablando para escuchar las intervenciones de los demás sin interrupciones de sonido.

- Confirmar la participación un día antes del encuentro, pues al tener un enfoque de aprendizaje colectivo, estos se abren cuando hay un mínimo confirmado de tres participantes.

\section{F. Público objetivo}

El trabajo de investigación que aquí se presenta está dirigido principalmente a educadores, investigadores, instituciones de educación pública y privada, academias de idiomas, líderes comunitarios, asociaciones civiles, y otras organizaciones interesadas en el desarrollo de círculos autogestivos para el aprendizaje de la lengua.

\section{DESCRIPCIÓN}

La metodología de la propuesta, orientada desde la perspectiva de género, permite a las participantes la oportunidad de posicionarse cómo agentes activas capaces de transformar sus realidades, a la vez que desarrollan sus estrategias y habilidades técnicas para el aprendizaje del idioma inglés.

\section{A. Diseño curricular de contenido y materiales}

Inspirado en el proyecto EDGE del British Council para la conformación de grupos autogestivos de mujeres para el desarrollo del liderazgo comunitario, el proyecto inició con un estudio de la literatura en torno a los temas de pedagogías populares, libertarias, descolonizadoras y feministas. Las principales aportaciones de la revisión bibliográfica incluyeron la inserción de conceptos y estrategias en torno a temas relevantes, como el papel de la lecto-escritura en procesos democratizadores (Freire, 2004), las ventajas y los obstáculos de la organización autogestiva para el aprendizaje a través de las experiencias de organizaciones sociales (Di Matteo, 2018) y la perspectiva de género en el desarrollo comunitario (Korol, 2007).

Una vez concluida la revisión de la literatura, se llevó a cabo la conformación del currículo del taller y los materiales didácticos. Este diseño curricular comenzó con la definición de una secuencia gramatical básica para convenir posteriormente con las participantes los temas y los materiales a utilizar. El currículum contempló estrategias didácticas típicas para el aprendizaje del idioma, tales como: repeticiones, juego de roles, lecturas de comprensión, etc. Estas estrategias didácticas fueron adaptadas a los temas de interés propuestos y se diseñó material para algunas etapas de la instrucción. Otros materiales relevantes fueron previstos para ser elaborados por las mismas participantes. Por otro lado, el currículum contempló la enseñanza de estrategias para el aprendizaje autogestivo del círculo a través de la asignación de tareas individuales y en equipos. Como parte de estas actividades, las participantes desarrollarían métodos específicos como el audio lingüístico o desarrollarían algunas estrategias específicas de lectura (skimming y scanning).

Sin embargo, a partir del surgimiento del Caso III, en un escenario tecnológico muy limitado, el currículum que había sido conformado en una primera etapa se tuvo que reestructurar por completo, para poder atender las necesidades específicas del grupo, a la vez que pudiera ser pertinente de acuerdo con los recursos disponibles. Esto ocasionó que se repensara el currículum en términos de un modelo flexible, adoptable por diferentes escenarios tecnológicos.

\section{A) Diseño de instrumentos técnico-metodológicos}

Debido a que el proyecto no cuenta con recursos económicos, más allá del trabajo voluntario de las tutoras e investigadores, 
el trabajo de campo se desarrolló a través del pilotaje de los cursos. Esto permitió ir adaptando las clases sin un currículum predeterminado, sino sujeto a las necesidades del círculo o grupo. En este sentido, tanto la infraestructura tecnológica (disponibilidad y acceso), como las necesidades e intereses de las participantes informan el proceso de planeación de sesiones y su evaluación.

Se diseñaron cuatro instrumentos para la recolección de datos a lo largo de los seis meses que duraron los talleres en su fase piloto, con la intención de evaluar el proceso:

\section{i) Pre-test}

El Pre-test se diseñó como un cuestionario, el cual fue aplicado a través de Forms de Google y se distribuyó a través del grupo de Whatsapp. El cuestionario funcionaba a su vez como registro de inscripción y estuvo dividido en tres secciones. En la primera de ellas, se solicitó información general sobre las participantes: edad, nacionalidad, ubicación, disponibilidad y acceso a tecnología, intereses en general y horarios de disponibilidad. En la segunda sección, se implementó un breve test de conocimientos del idioma inglés que nos permitió determinar su nivel de inglés. Por último, la tercera sección se componía de preguntas relacionadas con la gestión de su aprendizaje. En ella, se pidió a las participantes que definieran sus objetivos y/o intereses de aprendizaje y el tiempo semanal destinado al estudio del idioma.

ii) Entrevistas grupales semiestructuradas

Una vez transcurridos tres meses del taller, se llevó a cabo una entrevista grupal en cada uno de los grupos conformados (ocho en total). El propósito de estas entrevistas grupales semiestructuradas, consistió en conocer la perspectiva de las participantes, principalmente al respecto de las actividades y los materiales utilizados durante el taller; la encuesta contenía también una evaluación sobre el alcance de los objetivos del taller. La participación en la encuesta fue voluntaria y se dirigió a todas las participantes del círculo o grupo.

\section{iii) Post-test}

Al final del sexto mes, se llevó a cabo el post-test, a través del cual no solamente se verificaron los alcances de algunos objetivos de aprendizajes sobre la lengua inglesa, pero también se dio seguimiento a la calidad académica y ética del proyecto, permitiendo conocer los puntos de vista de las participantes al respecto de las actividades y los materiales utilizados durante el taller. La participación en la encuesta fue voluntaria y dirigida a todas las participantes de los círculos.

La fiabilidad del estudio, se garantiza en el cumplimiento de las expectativas de las alumnas. Es decir, dado que el cursotaller está diseñado para ellas, no requiere cumplir con una evaluación externa. Sin embargo, esto no significa que, para las participantes, la reflexión sobre su desempeño en lo individual $\mathrm{y}$ en relación con las demás fuese irrelevante, sino por el contrario. El proceso de auto y co-evaluación al final del curso, nos permite reconocer los aspectos a mejorar del programa para cumplir con las expectativas de las participantes.

\section{B) Implementación y seguimiento de talleres}

Los talleres se implementaron de enero a junio del 2021 tanto en México como en Colombia, y la distribución de horarios obedeció a las necesidades expresas de las participantes en cada caso. Incluso, las participantes decidieron los objetivos de las sesiones al solicitar sesiones orientadas al estudio específico de la gramática, sesiones de conversación o sesiones para actividades específicas, por ejemplo, la sesión de Karaoke virtual.

\section{C) Análisis de datos}

El análisis de los datos recolectados, se llevó a cabo con el fin de responder a cada una de las preguntas. En términos generales, sin embargo, se analizaron las respuestas de las participantes a través de un análisis cualitativo orientado a determinar temáticas de interés por separado en México y Colombia. Posteriormente, las tutoras en ambos países comentaron los resultados y los categorizaron.

\section{Resultados}

\section{A) Actividades y materiales}

El tipo de actividades y materiales que promovieron la autogestión del aprendizaje colectivo de las participantes (pregunta de investigación 1), fueron principalmente aquellas que cumplieron con las siguientes características: 1) fueron propuestas y gestionadas por las mismas participantes: por ejemplo, para la asignación de tareas de soporte técnico durante las lecciones o toma de notas para quienes no asistieron a la sesión; 2) los materiales también contribuyeron en este sentido y fueron adaptados de los diferentes materiales disponibles en internet: por ejemplo los planes de aprendizaje.

Además, las tutoras compartieron algunas de las estrategias didácticas utilizadas en sus grupos. Estas estrategias describían la consigna de la actividad, el tipo de tecnología y el material necesarios. El resultado fue la creación de una bitácora de materiales y actividades que pudieran ser utilizados o reutilizados.

En cuanto a los materiales, es preciso aclarar que se consideró la colaboración directa de las mismas participantes, hecho que resultó relevante para lograr un mayor compromiso para el trabajo colaborativo. Por ejemplo, una de las estrategias consistió en pedirles que conformaran un texto colectivo, integrado por preguntas en inglés orientadas a conocerse entre sí. Éstas preguntas, una vez validadas en conjunto con la tutora, fueron respondidas individualmente y las respuestas compartidas para la revisión colectiva.

\section{B) Pedagogías populares, descolonizadoras y feministas}

La respuesta a la segunda pregunta, pretende determinar las aportaciones de diversas pedagogías al desarrollo del trabajo autogestivo de aprendizaje colaborativo. En este sentido y como se definió anteriormente las aportaciones fueron principalmente en el sentido de construir un marco conceptual y didáctico que soportara la propuesta y fuera adaptable a las necesidades de cada contexto. En este documento no se profundiza en la respuesta a esta pregunta por cuestiones de alcances. Sin embargo, cabe mencionar que este tipo de pedagogías en general permitieron re conceptualizar la clase de idiomas en los roles de tutoras y estudiantes. Esto se logró a través del fomento de actividades donde se viera fortalecida la agencia de las participantes del círculo a través del trabajo colectivo y colaborativo. Esto, nos ha puesto en la disyuntiva de desarrollar las mismas fortalezas de liderazgo en las participantes que en las tutoras. Esto mismo, permitiría que en una segunda etapa del proyecto se pudiera pensar en la reproducción de estos círculos autogestivos de mujeres para el aprendizaje de la lengua inglés. 


\section{C) Uso de tecnología educativa}

La respuesta a la tercera pregunta sobre cuáles son los diferentes usos de la tecnología pertinentes para promover el aprendizaje colectivo de manera virtual, no es trivial. El factor definitivo será el acceso y la disponibilidad de infraestructura tecnológica.

Para el primer escenario tecnológico, en donde las participantes tuvieron disponibilidad y acceso a conexión de internet continua y uso de algunas plataformas gratuitas como Zoom o Google, los usos fueron tan variados como las opciones. Se utilizó software gratuito con diferentes finalidades. Por ejemplo, para la gestión de reuniones se utilizó Meets de Google y Zoom, en México para la gestión de los materiales, se utilizó Classroom. Para la presentación en las sesiones se utilizó Power Point, Jamboard y Drive de Google específicamente para la producción colaborativa y simultánea de documentos, presentaciones y tablas. Así mismo para la comunicación directa y avisos generales se utilizaron los grupos de Whatsapp, los cuales resultaron particularmente benéficos en el caso de escenarios con escasa disponibilidad tecnológica como se explicó anteriormente. Por otro lado, se promovió el uso de algunas aplicaciones gratuitas como CAKE. Por último, el internet fue una fuente fundamental de información para el diseño y adaptación de materiales didácticos y para la búsqueda de información por parte de las participantes.

Para el Caso III, ubicado en el segundo escenario tecnológico, previsto como un escenario de atención urgente, pero en donde se carecen de los recursos necesarios para la implementación completa del programa y se disponía únicamente de un móvil para todo el círculo, el uso de Whatsapp resultó fundamental. A través de esta plataforma tecnológica fue posible no solo compartir documentos en diferentes formatos aún, cuando las participantes no tenían correo electrónico, ni acceso continuo a internet. Por otro lado, aunque la comunicación oral a través de la llamada era continuamente deficiente, se sustituyó la llamada por el diseño de consignas precisas en español y en inglés grabadas y enviadas como mensajes de audio. Esto no sólo resolvió el problema de la comunicación oral, para el desarrollo de habilidades orales y de escucha, sino que además derivó en un uso que contempla adecuaciones metodológicas para el aprendizaje del idioma, como el desarrollo del método audio-lingüístico a través de algunas actividades.

\section{D) Fortalezas y debilidades del taller}

A partir de la aplicación de los tres instrumentos, fue posible determinar, en tres diferentes momentos de la implementación, las fortalezas y debilidades del taller. Con el pre-test, las participantes no sólo resolvieron un test de conocimientos de inglés para determinar su nivel, pero también proporcionaron información sobre los temas de interés y sus objetivos particulares de aprendizajes. Esta información permitió planear las lecciones y diseñar los materiales.

Posteriormente, a través de las entrevistas grupales, las participantes intervinieron continuamente para definir más temas y actividades de interés.

Por último, el post-test reveló el logro de los objetivos de aprendizaje de las estudiantes al mismo tiempo que señaló las principales características positivas del programa desde su percepción, así como las debilidades o aspectos a mejorar. A continuación, se resumen los principales hallazgos.
Los tres principales aspectos que más agradaron a las participantes de los círculos fueron: 1) la flexibilidad de los horarios; 2) la dinámica o interacción de las sesiones y 3) la atención de las tutoras. En segundo término, se mencionó el interés en los temas tratados, la paciencia de las tutoras, y el vocabulario aprendido. Por último, se mencionaron como aspectos más atractivos, aunque en menor número: la gratuidad del programa, los espacios para las conversaciones en inglés, la convivencia con otras mujeres y el acceso a materiales en formato de pdf y word. En estas respuestas, se demuestran varias razones por las que las participantes acudieron continuamente a las sesiones.

Sobre los temas a abordar en las sesiones, las respuestas fueron mucho más variadas de lo esperado de acuerdo con el pre-test. Sólo tres de los temas propuestos en esta ocasión se repitieron una vez. Estos temas fueron: ejercicios de comprensión oral, el aprendizaje de vocabulario a través del contexto, y los clubs de conversación. Otros temas a agregar: negocios, deportes, belleza, certificaciones, traducciones, evaluaciones, comprensión lectora, más canciones y más ejercicios. Cabe resaltar, que en el post-test, las respuestas estuvieron más orientadas a objetivos específicos de aprendizaje, que orientadas a temas generales de interés como durante el pre-test. Esto, a nuestro entender demuestra que las participantes han sido más precisas al determinar sus propios objetivos de aprendizaje; una habilidad para la autogestión.

En cuanto a los cambios que le harían al curso, el 50\% de quienes respondieron comentaron que ninguno. El 50\% restante opinó principalmente sobre el horario (33\%) dado que éste no siempre se ajusta a sus actividades; otras menciones incluyeron aumentar la disciplina, agregar ejercicios de escucha y más tareas y ejercicios. Quizás lo más interesante en este caso, es el $17 \%$ restante, cuyas respuestas se centran en el fortalecimiento de la acción o agencia de la tutora: llevar la disciplina del grupo, decidir sobre más ejercicios, etc. Esto demuestra, que los procesos de liderazgo que impulsan la autogestión, encuentran obstáculos conceptuales referentes a los roles de tutoras y participantes (aunque hayan sido establecidos y acordados con anterioridad).

En cuanto a la autoevaluación de su desempeño, 33\% de quienes respondieron a la encuesta dijeron tener un desempeño de su participación Bueno, el 50\% dijo que su desempeño fue Regular, y el $17 \%$ restante dijo haber tenido un desempeño Deficiente. Esto demuestra el nivel de auto evaluación, otra habilidad para el desarrollo autogestivo del círculo. Puede resumirse, que la mayoría de las participantes no está del todo satisfecha con su desempeño, lo que señala una necesidad de compromiso, vinculado a la motivación.

En cuanto a la coevaluación, un $42 \%$ de las participantes consideró que el desempeño de sus compañeras fue bueno, mientras que otro $42 \%$ lo consideró regular. Por otra parte, un $8 \%$ de las participantes consideró el desempeño de sus compañeras excelente, mientras el último $8 \%$ lo consideró deficiente. Estos resultados, a diferencia de los anteriores, muestra una distribución más homogénea en la apreciación del trabajo de las demás participantes.

Por último, en cuanto a la evaluación de las tutoras, el $83 \%$ de quienes respondieron que el desempeño de las tutoras fue excelente, mientras que el restante $17 \%$ opinó que fue bueno.

\section{CONCLUSIONES}


El proyecto FEM-CELL continuará al menos por un año más, sostenido a partir de los donativos de las participantes en esta segunda etapa y en búsqueda de financiamiento externo.

Para la planeación, organización y ejecución de la segunda etapa, los resultados hasta ahora obtenidos serán la base. A continuación, se mencionan las adecuaciones más importantes que deberán hacerse al programa (considerando que en la segunda etapa se escale su implementación), y las aportaciones del estudio realizado hasta ahora, argumentando las posibilidades de transferibilidad a otros contextos y las recomendaciones de aplicación.

1. Considerando el escalamiento del programa y generación de nuevos círculos, será necesario fortalecer la parte del currículo destinado al desarrollo de las habilidades autogestivas y agregar el desarrollo de habilidades de liderazgo. Esto, deberá acompañarse de un programa de formación de al menos 20 horas ( 2 horas semanales) para las nuevas tutoras. La propuesta plantea que las participantes de la primera etapa (piloto) podrán participar como tutoras para la segunda etapa.

2. Integración y redefinición de instrumentos de evaluación en México y Colombia. La experiencia del piloto permitirá rediseñar los instrumentos utilizados y agregar un breve informe mensual de seguimiento del trabajo tanto para las tutoras como para las estudiantes. El objetivo de este nuevo instrumento será facilitar el seguimiento y la evaluación del trabajo al interior de los círculos, tanto por parte de las participantes, como de las tutoras y la institución.

3. Otro instrumento necesario serán las observaciones de las sesiones, con el objetivo de poder triangular con los datos obtenidos a través de los demás instrumentos. Con esto, será necesario el desarrollo de nuevos instrumentos.

4. En cuanto a los materiales, se ha hecho imprescindible la elaboración de materiales en formato de texto (Word y Pdf) pero también en formato de audio y video. Así pues, el material deberá soportar la implementación de más actividades orientadas al desarrollo de habilidades para la comprensión auditiva, por ejemplo.

5. La tecnología deberá seguir explorándose en términos de objetivos de aprendizaje bien definidos, que justifiquen la pertinencia de los recursos tecnológicos y la capacitación en el uso de los mismos.

\section{AgRAdeCIMIENTOS}

Las autoras, autor e investigadores/as involucrados en este proyecto agradecemos a las participantes principalmente por la confianza depositada en este proyecto, y el apoyo de Hipatia México para su concreción.

\section{REFERENCIAS}

Argüello Cabrera, Libertad (2016). Homicidios, cotidianidad y la "Guerra contra el Narcotráfico". Atoyac de Álvarez, Guerrero (2007-2014). Iztapalapa Revista de Ciencias Sociales y Humanidades. núm. 87, año 40, (juliodiciembre de 2019) pp. 175-203. DOI: 10.28928/ri/872019/aot2/arguellocabreralsta

Arreola Medina, G. (2014). La Evaluación de la Calidad Educativa en México. Recuperado de: https://dialnet.unirioja.es/servlet/articulo? codigo=474339 6
British Council (2012) English and Digital for Girl's Education. Recuperado https://www.britishcouncil.org/society/womens-andgirls-empowerment/our-work/edge

Corvalán, G., Di Matteo, J., Downar, C., Guelman, A., Martínez Cajal, S., Palumbo, M. M., ...Vistosky, J. (2018). Pedagogías descolonizadoras: formación en el trabajo en los movimientos sociales. Ciudad Autónoma de Buenos Aires: CLACSO.

Di Matteo, J. (2018). Autogestión: una mirada desde la Educación Popular. A. Guelman y M. Mercedes Palumbo(Coord.). Pedagogías descolonizadoras: formación en el trabajo en los movimientos sociales ( $p p$. 65-88). Ciudad Autónoma de Buenos Aires: CLACSO.

Freire, P. (1972). Pedagogía del oprimido. Buenos Aires. Siglo XXI Argentina Editores

Freire, P. (2004). Cartas a quien pretende enseñar (2 reimpresión). Buenos Aires: Siglo XXI Editores Argentina.

Korol, C. (2007). Hacia una pedagogía feminista (1 ${ }^{\mathrm{a}}$ ed.). El Colectivo, América Libre.

Shinoda Bolen, J. (2008). El millonésimo círculo. Cómo transformarnos a nosotras mismas y al mundo. Barcelona. Editorial Kairós, S. A.

Solano López, A. y Trujillo Reyes, B. F. (2021). Hacer escuela entre silencios. Docentes de telesecundaria en contextos de narcotráfico. Revista Latinoamericana de Estudios Educativos. Volumen LI. Núm. 2 (mayo-agosto, 2021): 151-176. México D.F. SSN versión en línea: 2448-878X

Santiago, P., McGregor, I., Nusche, D., Ravela, P., \& Toledo, D. (2012). Revisiones de la OCDE sobre la Evaluación en Educación. MÉXICO. Recuperado de: https://www.oecd.org/education/school/RevisionesOCDE-evaluacion-educacion-Mexico.pdf 